\title{
Correction to: Predictive biomarkers and mechanisms underlying resistance to PD1/ PD-L1 blockade cancer immunotherapy
}

Daixi Ren ${ }^{1,2,3 \dagger}$, Yuze Hua ${ }^{1,2,3+}$, Boyao $\mathrm{Yu}^{1,2,3+}$, Xin Ye², Ziheng $\mathrm{He}^{2}$, Chunwei $\mathrm{Li}^{2}$, Jie Wang ${ }^{2}$, Yongzhen $\mathrm{Mo}^{2}$, Xiaoxu Wei', Yunhua Chen'², Yujuan Zhou', Qianjin Liao ${ }^{1}$, Hui Wang ${ }^{1}$, Bo Xiang ${ }^{1,2,3}$, Ming Zhou ${ }^{1,2,3}$, Xiaoling Li ${ }^{1,2,3}$, Guiyuan $\mathrm{Li}^{1,2,3}$, Yong $\mathrm{Li}^{4}$, Zhaoyang Zeng ${ }^{1,2,3}$ and Wei Xiong ${ }^{1,2,3^{*}}$

\section{Correction to: Molecular Cancer (2020) 19: 19}

\section{https://doi.org/10.1186/s12943-020-1144-6}

After publication of the article [1], authors found out that Fig. 1 was inadvertently replaced. Correct Fig. 1 version is provided on this paper.

The original article can be found online at https://doi.org/10.1186/s12943020-1144-6

* Correspondence: xiongwei@csu.edu.cn

${ }^{\dagger}$ Daixi Ren, Yuze Hua and Boyao Yu contributed equally to this work.

${ }^{1} \mathrm{NHC}$ Key Laboratory of Carcinogenesis and Hunan Key Laboratory of

Translational Radiation Oncology, Hunan Cancer Hospital and The Affiliated Cancer Hospital, Xiangya School of Medicine, Central South University, Changsha, Hunan, China

${ }^{2}$ Key Laboratory of Carcinogenesis and Cancer Invasion of the Chinese

Ministry of Education, Cancer Research Institute and School of Basic Medical

Science, Central South University, Changsha, Hunan, China

Full list of author information is available at the end of the article

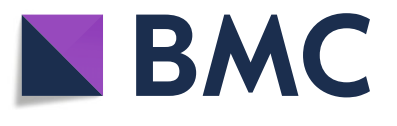

(c) The Author(s). 2020 Open Access This article is distributed under the terms of the Creative Commons Attribution 4.0 International License (http://creativecommons.org/licenses/by/4.0/), which permits unrestricted use, distribution, and reproduction in any medium, provided you give appropriate credit to the original author(s) and the source, provide a link to the Creative Commons license, and indicate if changes were made. The Creative Commons Public Domain Dedication waiver (http://creativecommons.org/publicdomain/zero/1.0/) applies to the data made available in this article, unless otherwise stated. 


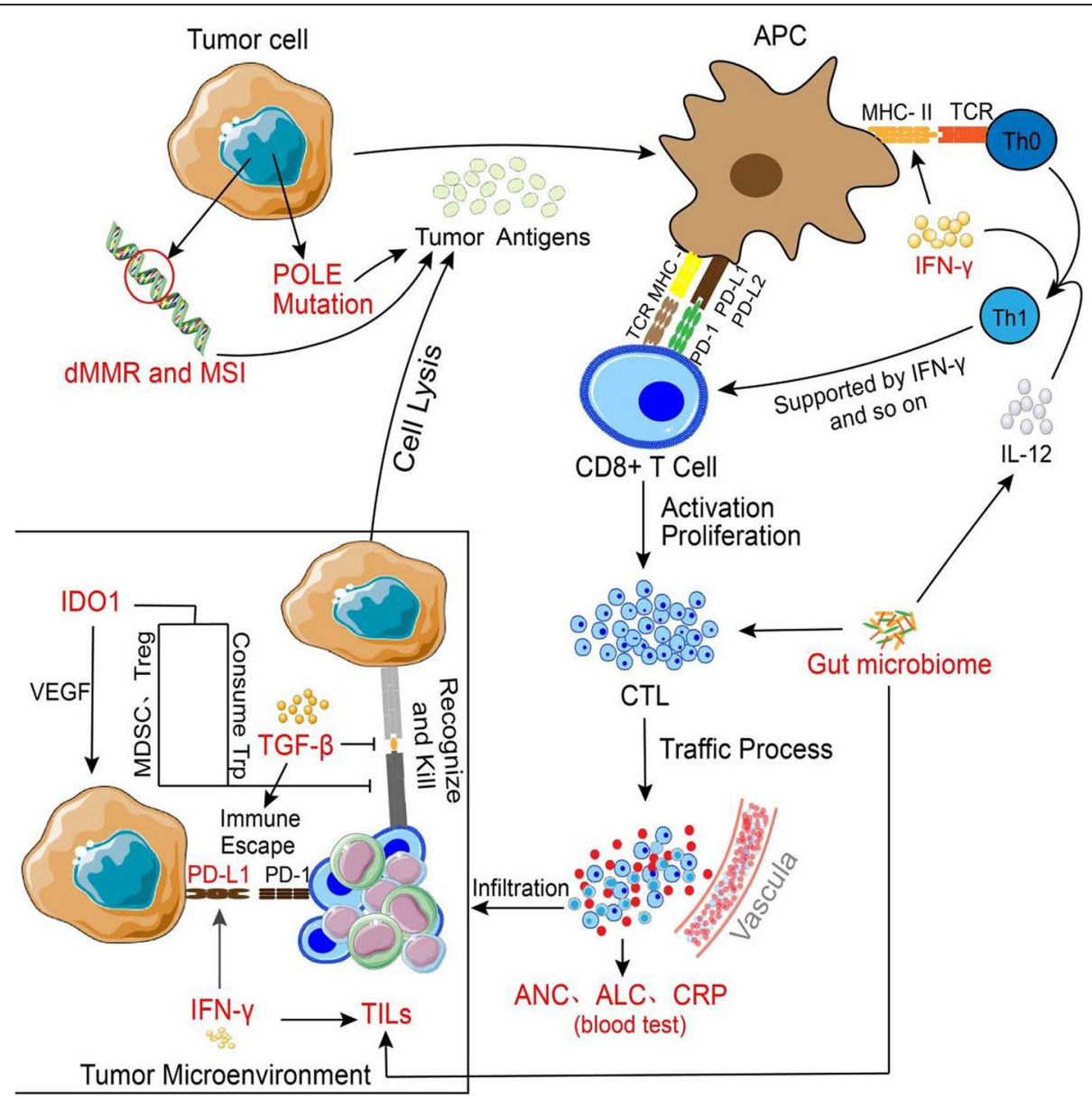

Fig. 1 Summary of biomarkers of the response to anti-PD-1/PD-L1 immunotherapy

\section{Author details}

${ }^{1} \mathrm{NHC}$ Key Laboratory of Carcinogenesis and Hunan Key Laboratory of Translational Radiation Oncology, Hunan Cancer Hospital and The Affiliated Cancer Hospital, Xiangya School of Medicine, Central South University, Changsha, Hunan, China. ${ }^{2}$ Key Laboratory of Carcinogenesis and Cancer Invasion of the Chinese Ministry of Education, Cancer Research Institute and School of Basic Medical Science, Central South University, Changsha, Hunan, China. ${ }^{3}$ Hunan Key Laboratory of Nonresolving Inflammation and Cancer, Disease Genome Research Center, The Third Xiangya Hospital, Central South University, Changsha, Hunan, China. ${ }^{4}$ Department of Medicine, Dan L Duncan Comprehensive Cancer Center, Baylor College of Medicine, Houston, TX, USA.

Published online: 14 February 2020

\section{Reference}

1. Ren D, Hua Y, Yu B, Ye X, He Z, Li C, Wang J, Mo Y, Wei X, Chen Y, Zhou Y, Liao Q, Wang H, Xiang B, Zhou M, Li X, Li G, Li Y, Zeng Z, Xiong W.

Predictive biomarkers and mechanisms underlying resistance to PD1/PD-L1 blockade cancer immunotherapy. Mol Cancer. 2020. https://doi.org/10.1186/ s12943-020-1144-6. 